\title{
Explicit inclusion of spin-orbit contribution in THSR wave function
}

\author{
N. Itagaki, ${ }^{1}$ H. Matsuno, ${ }^{2}$ and A. Tohsaki ${ }^{3}$ \\ ${ }^{1}$ Yukawa Institute for Theoretical Physics, Kyoto University, \\ Kitashirakawa Oiwake-Cho, Kyoto 606-8502, Japan \\ ${ }^{2}$ Department of Physics, Kyoto University, Kitashirakawa Oiwake-Cho, Kyoto 606-8502, Japan \\ ${ }^{3}$ Research Center for Nuclear Physics, 10-1 Mihogaoka, Ibaraki, Osaka 067-0047, Japan
}

(Dated: June 12, 2018)

\begin{abstract}
The Tohsaki-Horiuchi-Schuck-Röpke (THSR) wave function has been successfully used for the studies of gas-like nature of $\alpha$ clusters in various nuclei including the so-called Hoyle state of ${ }^{12} \mathrm{C}$ and four $\alpha$ states of ${ }^{16} \mathrm{O}$. In standard $\alpha$ cluster models, however, each $\alpha$ cluster wave function has spin zero because of its spatial symmetry and antisymmetrization effect. Thus the non-central interactions do not contribute, and this situation is the same in the THSR wave function. In this work, the spin-orbit contribution, which is found to be quite important at short $\alpha$ - $\alpha$ distances, is taken into account in the THSR wave function by combing it with antisymmetrized quasi cluster model (AQCM). The application to ${ }^{12} \mathrm{C}$ is presented. The multi-integration in the original THSR wave function is carried out by using Monte Carlo technique, which is called Monte Carlo THSR wave function. For the nucleon-nucleon interaction, the Tohsaki interaction, which contains finiterange three-body terms and simultaneously reproduces the saturation properties of nuclear systems, the $\alpha$ - $\alpha$ scattering phase shift, and the size and binding energy of ${ }^{4} \mathrm{He}$, is adopted.
\end{abstract}

\section{INTRODUCTION}

The Tohsaki-Horiuchi-Schuck-Röpke (THSR) wave function has been widely used for the studies of gas-like states of $\alpha$ clusters, including the so-called Hoyle state of ${ }^{12} \mathrm{C}$ and four $\alpha$ states of ${ }^{16} \mathrm{O}[1,2]$. In normal $\alpha$ cluster models, each $\alpha$ cluster is described by Gaussian-type wave function, and the positions of the $\alpha$ clusters are specified by Gaussian center parameters. In the THSR framework, all of these Gaussian center parameters are integrated out with a weight function, which enables us to describe well extended cluster states. Also, by choosing a small range for the weight function, the lowest configuration of the harmonic oscillator shell model is also described. Recently, "container picture" has been proposed for the description of nonlocalized clusters [3], and also, the framework is applied even to the nuclei such as ${ }^{9} \mathrm{Be} e t c$., which do not belong to $4 N$ nuclei $[4]$.

One of the problems of the traditional cluster models is that the non-central interactions, especially the spinorbit interaction, which is quite important in explaining the observed magic numbers, do not contribute; they work neither inside $\alpha$ clusters nor between $\alpha$ clusters. In cluster models, each $\alpha$ cluster is often defined as a simple $(0 s)^{4}$ configuration at some spatially localized point. In this case the antisymmetrization effect automatically makes the $\alpha$ cluster a spin singlet system free from the non-central interactions. To include the spin-orbit contribution starting with the cluster model, we proposed the antisymmetrized quasi cluster model (AQCM) [514]. This method allows us to smoothly transform $\alpha$ cluster model wave functions to $j j$-coupling shell model ones, and we call the clusters that feel the spin-orbit effect after this treatment quasi clusters. In AQCM, we have only two parameters: $R(\mathrm{fm})$ representing the distance between $\alpha$ clusters and $\Lambda(-)$ characterizing the transition of $\alpha$ cluster(s) to quasi cluster(s). It has been known that the conventional $\alpha$ cluster models cover the model space of closure of major shells $(N=2, N=8$, $N=20$, etc.) of the $j j$-coupling shell model. In addition, we have shown that the subclosure configurations of the $j j$-coupling shell model, $p_{3 / 2}(N=6), d_{5 / 2}(N=14)$, $f_{7 / 2}(N=28)$, and $g_{9 / 2}(N=50)$, where spin-orbit effect is quite important, can be reasonably described by our AQCM [1].

For such calculations, which include both cluster and shell features, we inevitably need a reliable nucleonnucleon interaction, not cluster-cluster interaction. It is quite well known that the central part of the interaction should have proper density dependence in order to satisfy the saturation property of nuclear systems. If we just introduce simple two-body interaction, for instance Volkov interaction [15], which has been widely used in the cluster studies, we have to properly choose Majorana exchange parameter for each nucleus, and consistent description of two different nuclei with the same Hamiltonian becomes almost impossible. Adding zero-range three-body interaction term helps better agreements with experiments; however the radius and binding energy of free ${ }^{4} \mathrm{He}(\alpha$ cluster $)$ are not well reproduced [16]. The Tohsaki interaction, which has finite range three-body terms, has much advantages [5, 17, 18]. Although this is a phenomenological interaction, it gives reasonable size and binding energy of the $\alpha$ cluster, and $\alpha-\alpha$ scattering phase shift is reproduced, while the saturation properties of nuclear matter is also reproduced rather sufficiently.

In this paper, we combine the THSR wave function and the idea of AQCM. It is worthwhile to show the applicability of the combined method by numerical calculations. The THSR wave function contains the multi-integration over the Gaussian center parameters of $\alpha$ clusters, and this procedure can be numerically performed using Monte Carlo technique called Monte Carlo THSR wave function [19 23]. In the present study, we calculate ${ }^{12} \mathrm{C}$ (three 
$\alpha)$ as the first step.

\section{FRAMEWORK}

In this section, we summarize the essence of the THSR wave function and AQCM, and combination of these two is newly introduced.

\section{A. Brink model}

The THSR wave function is based on the Brink model 24]. Each single particle wave function of the Brink model is described by a Gaussian,

$$
\phi_{i j}=\left(\frac{2 \nu}{\pi}\right)^{\frac{3}{4}} \exp \left[-\nu\left(\boldsymbol{r}_{i}-\boldsymbol{R}_{i}\right)^{2}\right] \chi_{j}
$$

where the Gaussian center parameter $\boldsymbol{R}_{i}$ shows the expectation value of the position of the $i$-th $\alpha$ cluster. The index $j$ specifies four nucleons in this $i$-th $\alpha$ cluster, and $\chi_{j}$ represents the spin isospin part of the single particle wave function. The size parameter $\nu$ is chosen to be $0.25 \mathrm{fm}^{-2}$, which reproduces the observed radius of ${ }^{4} \mathrm{He}$.

The Slater determinant of the Brink model is constructed from these single particle wave functions by antisymmetrizing them. Here, four single particle wave functions with different spin and isospin sharing a common Gaussian center parameter correspond to an $\alpha$ cluster.

$$
\begin{aligned}
\Phi_{S D}\left(\boldsymbol{R}_{1}, \boldsymbol{R}_{2}, \ldots, \boldsymbol{R}_{N}\right)= & \mathcal{A}\left\{\left(\phi_{11} \phi_{12} \phi_{13} \phi_{14}\right)\left(\phi_{21} \phi_{22} \phi_{23} \phi_{24}\right)\right. \\
& \left.\ldots\left(\phi_{N 1} \phi_{N 2} \phi_{N 3} \phi_{N 4}\right)\right\} .
\end{aligned}
$$

This is the case that we have $N \alpha$ clusters and the mass number $A$ is equal to $A=4 N$.

\section{B. THSR wave function}

The idea of the THSR wave function [1] is that Gaussian center parameters $\left\{\boldsymbol{R}_{i}\right\}$ are integrated over infinite space with the weight functions $\left\{\exp \left[-\boldsymbol{R}_{i}^{2} / \sigma^{2}\right]\right\}$. Thus the THSR wave function $\Phi_{T H S R}$ is expressed using $\Phi_{S D}$ in Eq. (2) as

$$
\begin{aligned}
\Phi_{T H S R}= & \int d \boldsymbol{R}_{1} d \boldsymbol{R}_{2} \cdots d \boldsymbol{R}_{N} \Phi_{S D}\left(\boldsymbol{R}_{1}, \boldsymbol{R}_{2}, \ldots, \boldsymbol{R}_{N}\right) \\
& \times \exp \left[-\left(\boldsymbol{R}_{1}^{2}+\boldsymbol{R}_{2}^{2} \cdots+\boldsymbol{R}_{N}^{2}\right) / \sigma^{2}\right]
\end{aligned}
$$

Here $\sigma$ is a control parameter, which governs the spatial extension of the system. When $\sigma$ is large, the wave function describes gas-like states of $\alpha$ clusters, and the lowest configuration of the harmonic oscillator shell model can be realized at the limit of $\sigma \rightarrow 0$.

\section{Monte Carlo THSR}

In some cases of the original THSR wave function, the analytic formula for the matrix elements for the Hamiltonian is already obtained. However for heavier nuclei, it is useful to introduce Monte Carlo technique for the multiintegration in the original THSR wave function [19 23]. We call this wave function $\left(\Phi_{M-T H S R}\right)$ Monte Carlo THSR,

$$
\Phi_{M-T H S R}=\sum_{k}^{N_{\max }} P^{J^{\pi}} \Phi_{S D}^{k}\left(\boldsymbol{R}_{1}, \boldsymbol{R}_{2}, \ldots, \boldsymbol{R}_{N}\right) .
$$

Here the multi-integration over the Gaussian center parameters in Eq. (3) is replaced with a summation of different Slater determinants. The Slater determinants superposed have different values of Gaussian center parameters $\left\{\boldsymbol{R}_{i}\right\}^{k}$ for $N \alpha$ clusters, where $k$ is a number to specify the set of the Gaussian center parameters for the $k$-th Slater determinant. The value of the Gaussian center parameters are randomly generated, but the distribution of the absolute value $\left|\boldsymbol{R}_{i}\right|$ for the $i$-th $\alpha$ cluster is introduced to be proportional to $\exp \left[-\boldsymbol{R}_{i}^{2} / \sigma^{2}\right]$, and its angular part is isotropically generated. Thus the information of the weight function in the original THSR wave function is absorbed in the distribution of randomly generated $\left\{\boldsymbol{R}_{i}\right\}^{k}$ values. The value of $N_{\max }$ shows the number of Slater determinants, which are superposed. The limit of $N_{\max } \rightarrow \infty$ coincides with the original THSR wave function; however we approximate it with a finite number. In Eq. (4), $P^{J^{\pi}}$ shows the projection onto the eigen states of parity and angular momentum, and this is numerically performed.

\section{AQCM}

In the conventional cluster models, there is no spinorbit effect for the $\alpha$ clusters. Thus they are changed into quasi clusters based on AQCM [5 14]. According to AQCM, when the original position of one of the $\alpha$ clusters (the value of Gaussian center parameter) is $\boldsymbol{R}$, the Gaussian center parameter of the $l$-th nucleon in this cluster is transformed by adding the imaginary part as

$$
\boldsymbol{\zeta}_{l}=\boldsymbol{R}+i \Lambda \boldsymbol{e}_{l}^{\mathrm{spin}} \times \boldsymbol{R}
$$

where $\boldsymbol{e}_{l}^{\text {spin }}$ is a unit vector for the intrinsic-spin orientation of the $l$-th nucleon in this $\alpha$ cluster. For the imaginary part, here we introduce $\Lambda$, which is a real dimensionless parameter. After this transformation, this $\alpha$ cluster is called quasi cluster. The imaginary part, which is added, depends on the spin direction of each nucleon, thus the $\alpha$ cluster is no longer a spin singlet system. The spin-orbit contribution can be taken into account by this transformation, and the contribution is attractive when $\Lambda$ is positive. We have previously shown that the lowest configurations of the $j j$-coupling shell model can be achieved by $\Lambda=1$ and $\boldsymbol{R} \rightarrow 0$. 


\section{E. Monte Carlo THSR+AQCM}

We propose a new framework by combining Monte Carlo THSR and AQCM, which is applied to ${ }^{12} \mathrm{C}$. For ${ }^{12} \mathrm{C}$, we introduce AQCM for all the three $\alpha$ clusters. Here the intrinsic-spins of the nucleons in the three $\alpha$ clusters are introduced to have threefold symmetry. This is needed to include the lowest configuration of the $j j$ coupling shell model (subclosure configuration of $p_{3 / 2}$ ) within a single Slater determinant. In the first $\alpha$ cluster, each intrinsic-spin of the four nucleons is spin-up $(z$ direction) for a proton and a neutron and spin-down $(-z$ direction) for a proton and a neutron. The intrinsic-spin orientations of the four nucleons in the second and third $\alpha$ clusters are introduced by rotating the ones of the first $\alpha$ cluster by $2 \pi / 3$ and $4 \pi / 3$ radians, respectively. These spin orientations of the twelve nucleons are fixed in all the Slater determinant before the angular momentum projection. While fixing the intrinsic-spin orientations, at first we randomly generate the Gaussian center parameters of three $\alpha$ clusters based on Monte Carlo THSR, and next, the center of mass of the total is shifted to the origin. Finally, imaginary parts of the Gaussian center parameters are introduced based on AQCM as in Eq. (5), and angular momentum projection and superposition of different Slater determinants follow. Here the second step of shifting the center of mass to the origin is quite important; the purpose of AQCM treatment is to describe the single particle motion of each nucleon around the origin and take into account the spin-orbit contribution, thus the center of the nucleus has to coincide with the origin of the coordinate system before giving the imaginary part.

\section{F. Hamiltonian}

The Hamiltonian $(\hat{H})$ consists of kinetic energy $(\hat{T})$ and potential energy $(\hat{V})$ terms, and the kinetic energy term is described as one-body operator,

$$
\hat{T}=\sum_{i} \hat{t}_{i}-T_{c m}
$$

and the center of mass kinetic energy $\left(T_{c m}\right)$, which is constant, is subtracted. The potential energy has central, spin-orbit, and the Coulomb parts. For the central part, we introduce the Tohsaki interaction [17], which has finite range three-body terms in addition to the twobody nucleon-nucleon interaction terms. This interaction is designed to reproduce both saturation property and the scattering phase shift of two $\alpha$ clusters. We use the F1 parameter set [17] in the present analysis, which was used in the original THSR work for ${ }^{12} \mathrm{C}$.

For the spin-orbit part $\left(\hat{V}_{s o}\right)$, the spin-orbit term of G3RS [25], which is a realistic interaction originally determined to reproduce the nucleon-nucleon scattering phase shift, is adopted;

$$
\hat{V}_{s o}=\sum_{i<j} V_{l s}\left(e^{-d_{1}\left(\vec{r}_{i}-\vec{r}_{j}\right)^{2}}-e^{-d_{2}\left(\vec{r}_{i}-\vec{r}_{j}\right)^{2}}\right) P\left({ }^{3} O\right) \vec{L} \cdot \vec{S},
$$

where $d_{1}=5.0 \mathrm{fm}^{-2}, d_{2}=2.778 \mathrm{fm}^{-2}$, and $P\left({ }^{3} O\right)$ is a projection operator onto a triplet odd state. The operator $\vec{L}$ stands for the relative angular momentum and $\vec{S}$ is the total spin, $\left(\vec{S}=\overrightarrow{S_{1}}+\overrightarrow{S_{2}}\right)$. The strength, $V_{l s}$, has been determined to reproduce the ${ }^{4} \mathrm{He}+n$ scattering phase shift [26], and $V_{l s}=1600-2000 \mathrm{MeV}$ has been suggested. Here we employ $V_{l s}=1800 \mathrm{MeV}$, which has been tested in our previous works for ${ }^{12} \mathrm{C}$ [5], although there the Majorana parameter for the three-body (central) interaction is slightly modified to reproduce the binding energy of ${ }^{16} \mathrm{O}$.

\section{RESULTS AND DISCUSSIONS}

We start Monte Carlo THSR calculation with the $\alpha$ non-breaking case, which is nothing but the superposition of Brink-type $\alpha$ cluster model wave functions. In the AQCM framework, this situation corresponds to setting $\Lambda$ in Eq. (5) to zero. In Fig. 1, the $0^{+}$energy convergence of ${ }^{12} \mathrm{C}$ (three $\alpha$ clusters) is shown as a number of Slater determinants superposed based on the Monte Carlo THSR framework ( $k$ in Eq. (41)). The solid, dotted, short-dashed, dashed, and dash-dotted lines correspond to $\sigma=1,2,3,4$, and $5 \mathrm{fm}$ in Eq. (3), respectively. The dashed line at $-82.50 \mathrm{MeV}$ shows the three $\alpha$ threshold energy. This is Monte Carlo calculation and not variational one, thus the energy is not always going down; the energy sometimes goes up with increasing number of the basis states. Nevertheless, the energy converges at the limit of $N_{\text {mas }} \rightarrow \infty$, but here we can confirm that it is well converged with 1000 basis states $\left(N_{\max }=1000\right.$ in Eq. (40). In this calculation, the radial part of the Gaussian center parameters $\left\{\boldsymbol{R}_{i}\right\}^{k}$ are generated by the random numbers $\left\{r_{i}\right\}$, whose distribution is proportional to $\exp \left[-r_{i}^{2} / \sigma^{2}\right]$, and the angular part of each $\boldsymbol{R}_{i}$ is isotropically generated using random numbers. It is found that the converged energy of small $\sigma$ case, $\sigma=1 \mathrm{fm}$ (solid line), is above the three $\alpha$ threshold. This is because the contribution of the spin-orbit interaction, which is important in inner regions, is missing within the $\Lambda=0$ wave functions. Other $\sigma$ values give the energies bellow the threshold, and the dotted line $(\sigma=2 \mathrm{fm})$ gives the lowest energy. With increasing the $\sigma$ value, the energy again goes up, and the energy of $\sigma=5 \mathrm{fm}$ (dash-dotted line) is close to the threshold.

Using these wave functions, the convergence of the root mean square (rms) matter radius for the $0^{+}$states of ${ }^{12} \mathrm{C}$ is shown in Fig. 2. The basis states are the same as those in Fig. 1, and wave functions are $\Lambda=0$ (Brink $\alpha$ cluster model), and the types of the lines are also the same; the solid, dotted, short-dashed, dashed, and dash-dotted lines correspond to $\sigma=1,2,3,4$ and $5 \mathrm{fm}$ in Eq. (3), 


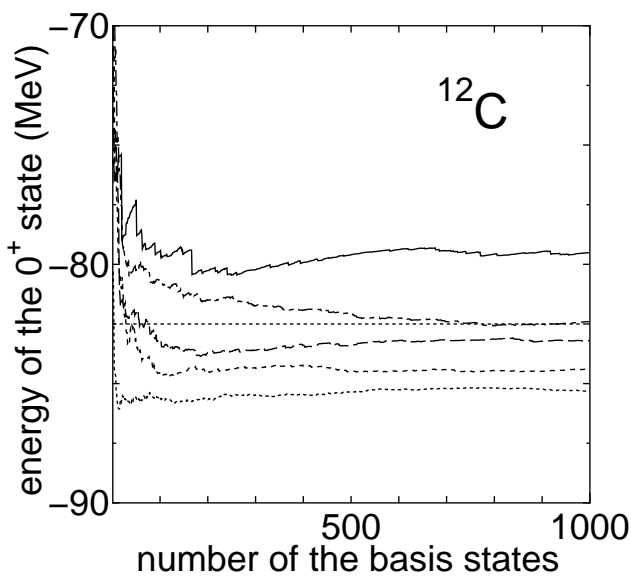

FIG. 1. The $0^{+}$energy convergence of ${ }^{12} \mathrm{C}$ (three $\alpha$ 's) as function of the number of Slater determinants superposed $(k$ in Eq. (4)) calculated with the Monte Carlo THSR framework. The $\alpha$ clusters are not broken $(\Lambda=0$ in Eq. (5) ), and the solid, dotted, dashed, short-dashed, dashed, and dash-dotted lines correspond to $\sigma=1,2,3,4$, and $5 \mathrm{fm}$ in Eq. (3), respectively. The dashed line at $-82.50 \mathrm{MeV}$ shows the three $\alpha$ threshold energy.

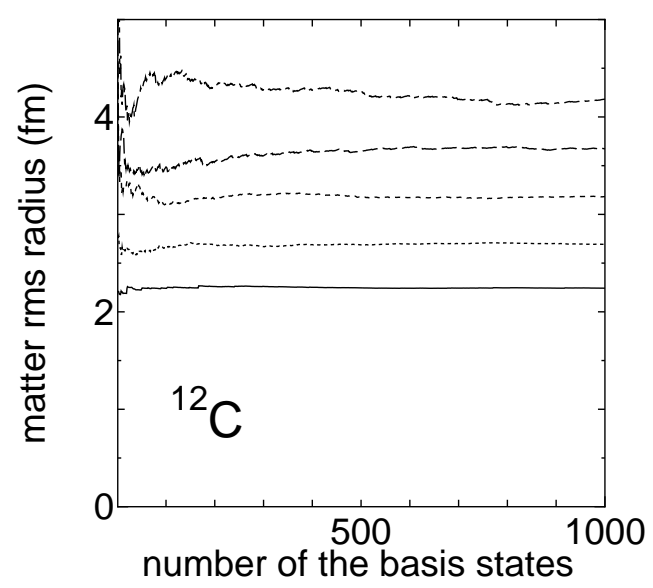

FIG. 2. The convergence of the root mean square (rms) matter radius for the $0^{+}$state of ${ }^{12} \mathrm{C}$ as a function of the number of Slater determinants superposed ( $k$ in Eq. (4) ) calculated with the Monte Carlo THSR framework. The $\alpha$ clusters are not broken ( $\Lambda$ in Eq. (5) is zero), and the solid, dotted, dashed, short-dashed, dashed, and dash-dotted lines correspond to $\sigma=1,2,3,4$, and $5 \mathrm{fm}$ in Eq. (3), respectively.

respectively. Experimentally the rms matter radius of ${ }^{12} \mathrm{C}$ is obtained as 2.35(2) fm in Ref. 27], consistent to the value deduced from the electron scattering, and the rms radius of $\sigma=1 \mathrm{fm}$ (solid line) is close to this value. If we compare the energy in Fig. 1 and the rms radius in Fig. 2. we find that this interaction gives the optimal state with slightly larger rms radius than experiments. However the energy curve with respect to the $\sigma$ value drastically changes if we incorporate the spin-orbit effect as we discuss shortly. For the second $0^{+}$state known as

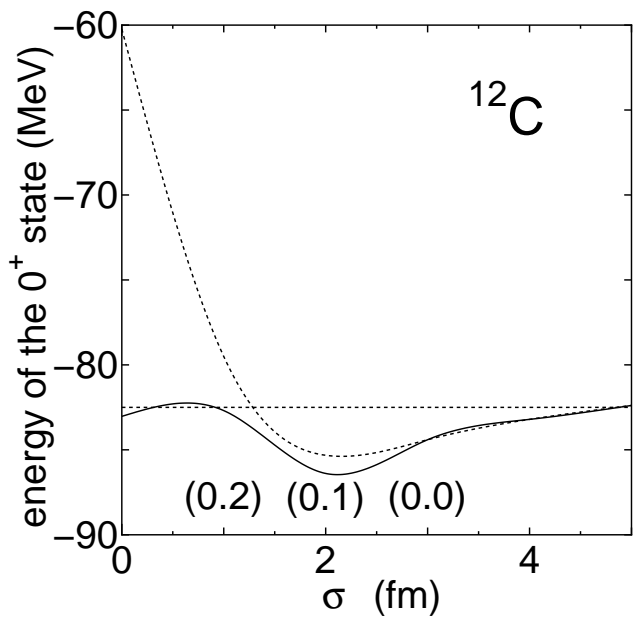

FIG. 3. The $0^{+}$energy curve of ${ }^{12} \mathrm{C}$ as a function of $\sigma$ (fm) defined in Eq. (3). The $\Lambda$ values defined in Eq. (5) is a variational parameter in the solid line, whereas $\Lambda$ is fixed to zero in the dotted line. For the solid line, the values in the parentheses show the optimal $\Lambda$ values for the cases of $\sigma=$ $1.0,2.0$, and $3.0 \mathrm{fm}$.

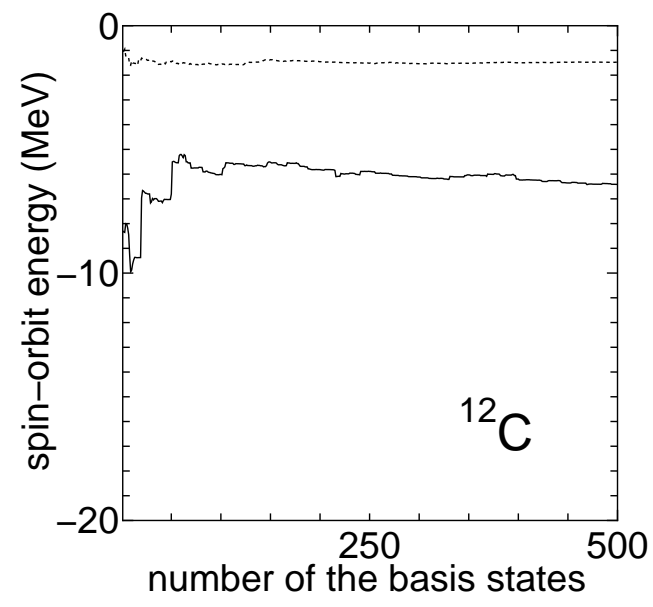

FIG. 4. The convergence of the spin-orbit energy for the $0^{+}$ state of ${ }^{12} \mathrm{C}$ as a function of number of Slater determinants superposed ( $k$ in Eq. (4) ). The solid line is for $\sigma=1.0 \mathrm{fm}$ and $\Lambda=0.2$, and the dotted line is for $\sigma=2.0 \mathrm{fm}$ and $\Lambda=0.1$.

the Hoyle state, the large rms radius of $\sim 4.0 \mathrm{fm}$ has been extensively discussed, although this state is a resonance state slightly above the threshold and the experimental determination is difficult. In the present case, the $\sigma=$ $5 \mathrm{fm}$ result (dash-dotted line) gives the energy around the threshold and the rms radius of $4.18 \mathrm{fm}$.

Then we take finite $\Lambda$ values, which allows us to take into account the spin-orbit contribution. The $0^{+}$energy curve for ${ }^{12} \mathrm{C}$ is shown in Fig. 3 as a function of $\sigma(\mathrm{fm})$ defined in Eq. (3). The $\Lambda$ values defined in Eq. (5) is a variational parameter in the solid line, whereas $\Lambda$ is fixed to zero in the dotted line. For the solid line, the values in the parentheses show the optimal $\Lambda$ values for 
the cases of $\sigma=1.0,2.0$, and $3.0 \mathrm{fm}$. We can see large decrease of the energy by more than $20 \mathrm{MeV}$ at the limit of $\sigma=0 \mathrm{fm}$ after optimizing the $\Lambda$ value. Then in the case of the solid line, a local minimum point appears at the limit of $\sigma=0 \mathrm{fm}$, where the contribution of the spin-orbit interaction is very large, more than $-30 \mathrm{MeV}$. The optimal $\Lambda$ value of 0.3 at the limit of $\sigma=0 \mathrm{fm}$ shows that $\alpha$ clusters are broken to some extent and the wave function approaches to the $j j$-coupling shell model one. In general, the contribution of the spin-orbit interaction increases and the energy decreases with increasing $\Lambda$ (for each fixed $\sigma$ ), but it saturates at some point. On the other hand, the kinetic energy quadratically increases with increasing $\Lambda$, and the energy minimum state appears owing to the compensation of these two factors.

As a function of $\sigma$, in Fig. 3, the energy minimum point of the solid line appears around $\sigma=2.0 \mathrm{fm}$, where the optimal $\Lambda$ value is 0.1 . Owing to the additional attraction of the spin-orbit interaction, here the solid line is lower than the dotted line by about $1 \mathrm{MeV}$. If we mix two minimum points, the true minimum point of $(\sigma \mathrm{fm}, \Lambda)=(2.0 \mathrm{fm}, 0.1)$ and the local minimum point of $(\sigma \mathrm{fm}, \Lambda)=(0.0 \mathrm{fm}, 0.3)$, with the amplitude ratio of 2:1 after normalizing each of these two, the rms matter radius becomes $2.37 \mathrm{fm}$, which reproduces the experimental value.

The convergence of the spin-orbit energy for the $0^{+}$ state of ${ }^{12} \mathrm{C}$ is shown in Fig. 4 as a function of number of Slater determinants superposed ( $k$ in Eq. (4) ). This is a demonstration that the spin-orbit effect can be successfully taken into account with the procedure proposed here. The solid line is for $\sigma=1.0 \mathrm{fm}$ and $\Lambda=0.2$, and the dotted line is for $\sigma=2.0 \mathrm{fm}$ and $\Lambda=0.1$. The $\Lambda$ values are optimal ones in each $\sigma$ case, and the latter gives the lowest energy point in Fig. [4 The solid line $(\sigma \mathrm{fm}, \Lambda)=(1.0 \mathrm{fm}, 0.2)$ converges to $\sim-6.4 \mathrm{MeV}$, whereas the dotted line $(\sigma \mathrm{fm}, \Lambda)=(2.0 \mathrm{fm}, 0.1)$ converges to $\sim-1.5 \mathrm{MeV}$.

After introducing the finite value of the $\Lambda$ values, each nucleon is more independently treated and calculation costs significantly increases compared with the Brink model $(\Lambda=0)$ calculation. Therefore, in Fig. 3, although the contribution of the kinetic energy is calculated with superposing 1000 Slater determinants $\left(N_{\max }=1000\right.$ in Eq. (4)), the contribution of the two-body interactions is estimated with 500 Slater determinants $\left(N_{\max }=500\right)$. The most time consuming part is the finite-range threebody interaction part. This three-body part is substituted with the values obtained with the $\Lambda=0$ wave functions. The three-body interaction terms do not strongly depend on the $\Lambda$ values, and we can approximate it with the Brink model. The Brink model calculation is rather simple and we can superpose 1000 basis states for the estimation of the three-body terms. This approximation can be justified in Fig. 5 , which shows the contribution of the three-body interaction. The $\sigma$ value is $2.0 \mathrm{fm}$, and the solid line is for $\Lambda=0.1$, which gives the optimal energy in Fig. 3 and the dotted line is for $\Lambda=0.0$. The

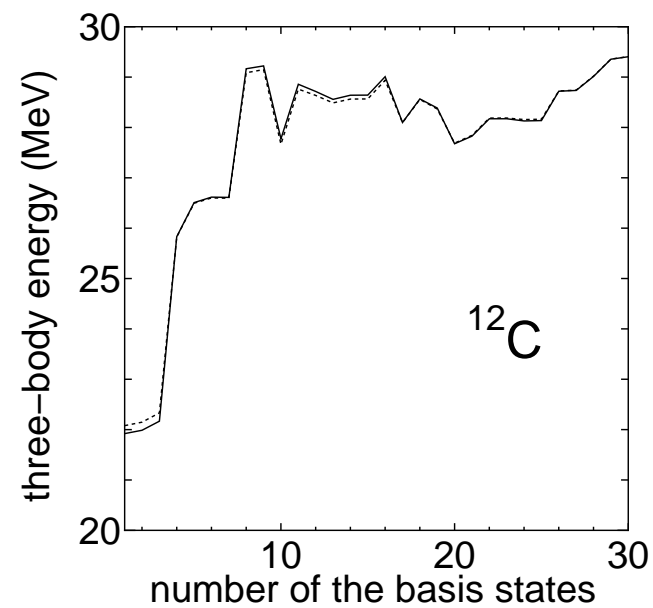

FIG. 5. The contribution of three-body interaction terms for the $0^{+}$state of ${ }^{12} \mathrm{C}$ as a function of the number of Slater determinants superposed ( $k$ in Eq. (4) ) calculated with the Monte Carlo THSR framework. The $\sigma(\mathrm{fm})$ value defined in Eq. (3) is $2.0 \mathrm{fm}$, and $\Lambda$ in Eq. (5) is 0.1 (solid line) and 0 (dotted line).

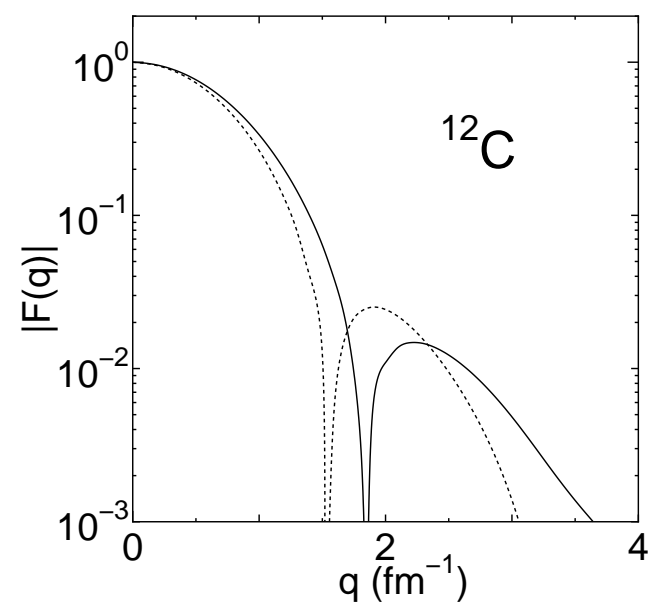

FIG. 6. The absolute value of the elastic form factor $\left.\left(\mid F_{(} q\right) \mid\right)$ for the $0^{+}$state of ${ }^{12} \mathrm{C}$ as a function of the momentum transfer $q\left(\mathrm{fm}^{-1}\right)$. The dotted line is for $(\sigma \mathrm{fm}, \Lambda)=(2.0 \mathrm{fm}, 0.1)$, which gives the optimal energy as shown in Fig. 3 and the solid line is the result after mixing the local minimum point (at the limit of $\sigma=0 \mathrm{fm}$ limit, $\Lambda=0.3$ ) with the amplitude ratio of $2: 1$. The experimental values and other theoretical results are compared in Refs. [28, 29].

real parts of Gaussian center parameters for each Slater determinant are common for the $\Lambda=0.0$ (dotted line) and $\Lambda=0.1$ (solid line) cases, and imaginary parts are just added to the real parts in the $\Lambda=0.1$ case following Eq. (5). The energies are not converged yet within such a small number of the basis states, but the values of these two lines for the three-body interaction terms are very close not to be distinguished. Indeed, the difference is less than $100 \mathrm{keV}$. Then we can estimate the contribution 
of three-body terms in the finite $\Lambda$ cases by superposing Brink-type Slater determinants $(\Lambda=0)$, where number of the basis states $\left(N_{\max }\right)$ is not 30 as in this figure but increased to 1000 .

The absolute value of the elastic form factor $\left(\left|F_{(q)}\right|\right)$ for the $0^{+}$state of ${ }^{12} \mathrm{C}$ is shown in Fig. 6 as a function of the momentum transfer $q\left(\mathrm{fm}^{-1}\right)$. The dotted line is for $(\sigma \mathrm{fm}, \Lambda)=(2.0 \mathrm{fm}, 0.1)$, which gives the optimal energy as shown in Fig. 3 The solid line is the result after mixing the local minimum point (at the limit of $\sigma=0 \mathrm{fm}$, $\Lambda=0.3)$. The mixing ratio of $(\sigma=2.0 \mathrm{fm}, \Lambda=0.1)$ : $(\sigma=0 \mathrm{fm}, \Lambda=0.3)$ is given as 2:1 after normalizing each configuration. The experimental values and other theoretical results are compared in Refs. [28, 29]. The dotted line line shows the sign change around $q \sim 1.6 \mathrm{fm}^{-1}$, which is too small compared with the experiment, reflecting the fact that the $(\sigma \mathrm{fm}, \Lambda)=(2.0 \mathrm{fm}, 0.1)$ configuration, which is energetically optimal one, has too large spacial extension compared with the experiment (experimentally this sign change occurs around $q \sim 1.8 \mathrm{fm}^{-1}$ ). We have previously mentioned that the mixing of two states, the energy optimal one and the local minimum point with smaller radius, enables us to reproduce the experimental rms radius, and this mixing turns out to be also important in reproducing the form factor, which is the solid line.

\section{SUMMARY}

The THSR wave function has been successfully used for the studies of gas-like nature of $\alpha$ clusters of various nuclei. In this work, we proposed a method to take into account the spin-orbit contribution in THSR by combing it with AQCM. In the standard $\alpha$ cluster models, each $\alpha$ cluster wave function has spin zero because of the spatial symmetry of the $\alpha$ clusters and antisymmetrization effect. Thus the non-central interactions do not contribute, and this situation is the same in the THSR wave function. The application of a new framework to ${ }^{12} \mathrm{C}$ was presented. The multi-integration in the original THSR wave function was carried out by using Monte Carlo technique, which is called Monte Carlo THSR wave function. In ${ }^{12} \mathrm{C}$, the contribution of the spin-orbit interaction was successfully taken into account. Especially for the cases when the spatial extension is small, the contribution is quite strong, but it decreases with increasing the spatial extension. As a result, one local minimum at the limit of zero distance between $\alpha$ clusters and the real minimum state with sizable $\alpha-\alpha$ distances appear. If we mix these two configurations, we can reproduce the observed matter rms radius. This can be considered as the quantum mechanical mixing of different structures, or more concretely, competition of shell and cluster structures.

As a future work, we further apply the combined framework of Monte Carlo THSR and AQCM, which was proposed in the present study, to other cases. Also, we try to derive analytic formula for the matrix elements of the Hamiltonian for the combined framework.

\section{ACKNOWLEDGMENTS}

Numerical calculation has been performed using the computer facility of Yukawa Institute for Theoretical Physics, Kyoto University. This work was supported by JSPS KAKENHI Grant Number 17K05440.
[1] A. Tohsaki, H. Horiuchi, P. Schuck, and G. Röpke, Phys. Rev. Lett. 87, 192501 (2001).

[2] Y. Funaki, H. Horiuchi, and A. Tohsaki, Progress in Particle and Nuclear Physics 82, 78 (2015)

[3] B. Zhou, Y. Funaki, H. Horiuchi, Z. Ren, G. Röpke, P. Schuck, A. Tohsaki, C. Xu, and T. Yamada, Phys. Rev. Lett. 110, 262501 (2013).

[4] M. Lyu, Z. Ren, B. Zhou, Y. Funaki, H. Horiuchi, G. Röpke, P. Schuck, A. Tohsaki, C. Xu, and T. Yamada, Phys. Rev. C 91, 014313 (2015)

[5] N. Itagaki, Phys. Rev. C 94, 064324 (2016)

[6] N. Itagaki, H. Masui, M. Ito, S. Aoyama, and K. Ikeda, Phys. Rev. C 73, 034310 (2006).

[7] H. Masui No and Nagaki, Phys. Rev. C 75, 054309 (2007).

[8] T. Yoshida, N. Itagaki, and T. Otsuka, Phys. Rev. C 79, 034308 (2009)

[9] N. Itagaki, J. Cseh, and M. Płoszajczak, Phys. Rev. C 83, 014302 (2011).

[10] T. Suhara, N. Itagaki, J. Cseh, and M. Płoszajczak, Phys. Rev. C 87, 054334 (2013)

[11] N. Itagaki, H. Matsuno, and T. Suhara, Progress of The- oretical and Experimental Physics 2016, 093 D01 (2016).

[12] H. Matsuno, N. Itagaki, T. Ichikawa, Y. Yoshida, and Y. Kanada-Enyo, Progress of Theoretical and Experimental Physics 2017, 063D01 (2017).

[13] H. Matsuno and N. Itagaki, Progress of Theoretical and Experimental Physics 2017, 123D05 (2017).

[14] N. Itagaki and A. Tohsaki, Phys. Rev. C 97, 014307 (2018).

[15] A. Volkov, Nuclear Physics 74, 33 (1965)

[16] T. Ando, K. Ikeda, and A. Tohsaki-Suzuki, Progress of Theoretical Physics 64, 1608 (1980).

[17] A. Tohsaki, Phys. Rev. C 49, 1814 (1994)

[18] N. Itagaki, A. Ohnishi, and K. Kato, Progress of Theoretical Physics 94, 1019 (1995)

[19] N. Itagaki, M. Kimura, C. Kurokawa, M. Ito, and W. von Oertzen, Phys. Rev. C 75, 037303 (2007).

[20] N. Itagaki, T. Kokalova, M. Ito, M. Kimura, and W. von Oertzen, Phys. Rev. C 77, 037301 (2008).

[21] N. Itagaki, M. Ito, K. Arai, S. Aoyama, and T. Kokalova, Phys. Rev. C 78, 017306 (2008).

[22] S. Aoyama and N. Itagaki, Phys. Rev. C 80, 021304 (2009). 
[23] N. Itagaki, T. Kokalova, and W. von Oertzen, Phys. Rev. C 82, 014312 (2010)

[24] D. M. Brink, Proc. Int. School Phys. "Enrico Fermi" XXXVI, 247 (1966).

[25] R. Tamagaki, Progress of Theoretical Physics 39, 91 (1968).

[26] S. Okabe, Y. Abe, and H. Tanaka, Progress of Theoretical Physics 57, 866 (1977).

[27] R. Kanungo, W. Horiuchi, G. Hagen, G. R. Jansen, P. Navratil, F. Ameil, J. Atkinson, Y. Ayyad, D. CortinaGil, I. Dillmann, A. Estradé, A. Evdokimov, F. Farinon, H. Geissel, G. Guastalla, R. Janik, M. Kimura,
R. Knöbel, J. Kurcewicz, Y. A. Litvinov, M. Marta, M. Mostazo, I. Mukha, C. Nociforo, H. J. Ong, S. Pietri, A. Prochazka, C. Scheidenberger, B. Sitar, P. Strmen, Y. Suzuki, M. Takechi, J. Tanaka, I. Tanihata, S. Terashima, J. Vargas, H. Weick, and J. S. Winfield, Phys. Rev. Lett. 117, 102501 (2016)

[28] Y. Fujiwara, H. Horiuchi, K. Ikeda, M. Kamimura, K. Katō, Y. Suzuki, and E. Uegaki, Progress of Theoretical Physics Supplement 68, 29 (1980).

[29] A. Lovato, S. Gandolfi, R. Butler, J. Carlson, E. Lusk, S. C. Pieper, and R. Schiavilla, Phys. Rev. Lett. 111, 092501 (2013) 